\title{
Selected Metal Matrix Metalloproteinases and Tissue Inhibitors of Metalloproteinases as Potential Biomarkers for Tubulointerstitial Fibrosis in Children with Unilateral Hydronephrosis
}

\author{
Beata Bieniaś (DD and Przemysław Sikora \\ Department of Pediatric Nephrology Medical University of Lublin, Gębali 6, 20-093 Lublin, Poland \\ Correspondence should be addressed to Beata Bieniaś; beata.bienias@umlub.pl
}

Received 29 December 2019; Revised 2 June 2020; Accepted 17 June 2020; Published 29 June 2020

Academic Editor: Hubertus Himmerich

Copyright (c) 2020 Beata Bieniaś and Przemysław Sikora. This is an open access article distributed under the Creative Commons Attribution License, which permits unrestricted use, distribution, and reproduction in any medium, provided the original work is properly cited.

\begin{abstract}
Renal tubulointerstitial fibrosis caused by congenital ureteropelvic junction obstruction (UPJO) may lead to the development of obstructive nephropathy $(\mathrm{ON})$ and the impairment of kidney function. Hence, the identification of early biomarkers of this condition might be of assistance in therapeutic decisions. This study evaluates serum and urinary metalloproteinases MMP-1, MMP-2, and MMP-9 and tissue inhibitors of metalloproteinases TIMP-1 and TIMP-2 as potential biomarkers of ON in children with congenital unilateral hydronephrosis $(\mathrm{HN})$ caused by UPJO. Forty-five (45) children with congenital HN of different grades of severity and twenty-one (21) healthy controls were enrolled in the study. Urinary and serum concentrations of MMP-1, MMP-2, MMP-9, TIMP-1 and TIMP-2 were measured using specific ELISA kits. The urinary excretions were expressed as biomarker/creatinine ( $\mathrm{Cr}$ ) ratios. To evaluate the extracellular matrix remodelling process activity, the serum and urinary MMP-1, -2, -9/TIMP-1, -2 ratios were also calculated. In comparison with the controls, patients with HN, independent of the grade, showed significantly increased median serum MMP-9, TIMP-1, and TIMP-2, median urinary MMP-9/Cr, and TIMP-2/Cr ratios. Lower median values of serum MMP-2/TIMP-1, MMP-9/TIMP-1 in patients with HN were also revealed. Additionally, higher urinary MMP-2/Cr, lower urinary MMP-2/TIMP-2, and lower serum MMP-9/TIMP-2 ratios were observed in patients with HN grades 3 and 4. Patients with ON diagnosed by renal scintigraphy had a significantly higher median serum MMP-9 concentration and lower median serum MMP-9/TIMP-1, -2 ratios in comparison with those without this condition. Patients with nonglomerular proteinuria had a significantly higher median serum TIMP-1 concentration, a higher median urinary TIMP-2/Cr ratio, and a lower serum MMP-9/TIMP-1 ratio compared to those without this symptom. The relationship between the measured biomarkers and the relative function of the obstructed kidney showed no correlations. The ROC curve analysis showed a promising diagnostic profile for the detection of ON for serum MMP-9 and the serum MMP-9/TIMP-1 and MMP-9/TIMP-2 ratios. In conclusion, the results of this study suggest that patients with HN, particularly with grades 3 and 4, are at higher risk of renal tubulointerstitial fibrosis. The noninvasive markers of this condition considered are urinary MMP-2/Cr and MMP-9/Cr, serum MMP-9, serum and urinary MMP-2, MMP-9/TIMP-1, -2. Additionally, serum MMP-9 and MMP-9/TIMP-1, -2 may become promising markers of $\mathrm{ON}$.
\end{abstract}

\section{Introduction}

Obstructive nephropathy (ON) caused by congenital ureteropelvic junction obstruction (UPJO) is progressive tubulointerstitial fibrosis leading to glomerular sclerosis and the impairment of kidney function $[1,2]$. The pathogenesis of renal fibrosis is complex. The final stages of its development are related to an imbalance between the formation and degradation of the extracellular matrix (ECM).

Multiple factors such as the grade of hydronephrosis, urinary tract infections, and individual considerations are the sole determinants of the mechanism of renal fibrosis and its initiation and progression. Therefore, identification of early biomarkers of this condition might help in better 
TABLE 1: Characteristics of study and control groups.

\begin{tabular}{lcc}
\hline Parameter & Study group median (range) and number of patients & Control group \\
\hline Number of patients & 45 & 21 \\
Gender (male/female) & $31 / 14$ & $16 / 5$ \\
Age (years) & $11.0(2-17)$ & $12.3(3-17)$ \\
GFR $\left(\mathrm{ml} / \mathrm{min} / 1.73 \mathrm{~m}^{2}\right)$ & $126.3(97-162)$ & $139(102-145)$ \\
Number of patients in groups A/B/C & $25 / 11 / 9$ & - \\
Number of patients with obstructive nephropathy & $28 / 45(62.2 \%)$ & - \\
Number of patients with proteinuria & $10 / 45(22.2 \%)$ & - \\
Protein/creatinine ratio $(\mathrm{mg} / \mathrm{mg})$ & $0.24(0.21-0.4)$ & $0.09(0-0.15)$ \\
\hline
\end{tabular}

stratification of patients with UPJO and facilitate therapeutic decisions.

In the recent years, matrix metalloproteinases (MMPs), which are proteolytic enzymes that degrade matrix proteins [3] and their tissue inhibitors (TIMPs), have been studied as a potential marker of renal fibrosis.

MMPs are a large family of zinc-containing matrix degrading enzymes including mainly collagenases and stromelysins [4-6]. Recent studies show that MMPs may be implicated in the initiation and progression of kidney fibrosis and the development of chronic kidney disease (CKD) $[4,7-$ 9]. MMP-1, MMP-2, and MMP-9 and TIMP-1 and TIMP-2 contribute to the ECM remodelling processes within the renal interstitium $[10,11]$.

The gelatinases MMP-2 and MMP-9 cleave denatured collagen, type IV collagen, and laminin [12] with both expressed in different renal structures, including the glomeruli, the proximal and distal tubules, and the collecting ducts [12-17]. They are also pivotal to the recruitment and chemotaxis of inflammatory cells [18]. MMP-2 is also the potential activator of MMP-1 and MMP-9 by cleaving their prodomains [19]. It was observed that MMP-9 contributes to the pathogenesis of renal fibrosis through macrophage recruitment and osteopontin cleavage [20]. Similar to MMP-2, it may induce tubular cell epithelial-mesenchymal transition $[20,21]$. In addition, MMP- 2 can promote ECM production and accumulation [22]. There is also a positive correlation between the urinary excretions of MMP-2 and MMP-9 and transforming growth factor beta (TGF-beta), confirming their profibrotic action [23]. MMP-1 is interstitial collagenase, which degrades the native collagen and is hypothesised to be an antifibrotic enzyme [24, 25]. However, its role in kidney diseases remains incompletely understood.

"In vitro" and animal studies show a higher activity of MMPs and TIMPs in the process of kidney fibrosis [7, 24, 25]. Several clinical studies demonstrated increased serum levels and/or urinary excretions of these MMPs and their inhibitors TIMP- 1 and TIMP-2 in patients with CKD making them promising markers for this condition $[23,26]$. However, to the best of our knowledge, there are few reports on the evaluation of MMPs and TIMPs in HN.

\section{Purpose of the Study}

The purpose of this study is to evaluate the serum and urinary metalloproteinases MMP-1, MMP-2, and MMP-9 and the tissue inhibitors of metalloproteinases TIMP-1 and TIMP-2 as potential biomarkers of $\mathrm{ON}$ in children with congenital unilateral hydronephrosis (HN). These parameters were evaluated in relation to the severity of $\mathrm{HN}$, the presence of signs of $\mathrm{ON}$ in renal scintigraphy, the relative function of an obstructed kidney, and the presence of proteinuria.

\section{Patients}

Table 1 shows the baseline characteristics of patients and controls. The study comprised 45 children ( 31 boys and 14 girls) aged 2-17 years (median, 11.0 years) with congenital unilateral $\mathrm{HN}$ secondary to UPJO, diagnosed and treated in our department. Using the Onen HN ultrasound grading system [27], HN was classified as follows: stage 1: solitary dilatation of the renal pelvis; stage 2: the same as stage 1 including dilatation of the renal calices; stage 3: the same as stage 2 including $<1 / 2$ (mild-to-moderate) renal parenchymal loss; and finally, stage 4 : the same as stage 3 plus $>1 / 2$ (severe) renal parenchymal loss (cyst-like kidney with no visually significant renal parenchyma). Accordingly, the patients were divided into three subgroups: A, B, and C. Of the 45 children, 25 (55.6\%) with $\mathrm{HN}$ grades 3 and 4 were included in group A, with 11 (24.4\%) with HN grade 2 in group B and 9 (20\%) with $\mathrm{HN}$ grade 1 in group C.

Dynamic renal scintigraphy using Technetium-99m-L ethylenedicysteine was performed in all patients to determine signs of ON defined as renal parenchymal defects with decreased relative function of the obstructed kidney. Accordingly, ON was revealed in 28/45 (62.2\%) patients. There were 21 patients from group A and 7 from group B. The split function of the obstructed kidney compared to the normal kidney ranged from 15 to $85 \%$ and 35 to $65 \%$.

Pathological, nonglomerular proteinuria, as an indication of tubular injury (urinary protein/creatinine ratio: $>0.21 \mathrm{mg} / \mathrm{mg}$, median: $0.24 \mathrm{mg} / \mathrm{mg}$, range: $0.21-0.4 \mathrm{mg} / \mathrm{mg}$ ), was revealed in 10 patients. All were from group A, and 7 had signs of ON.

All patients had a normal estimated glomerular filtration rate (eGFR) $>90 \mathrm{ml} / \mathrm{min} / 1.73 \mathrm{~m}^{2}$ calculated by the Schwartz formula [28]. Twenty-one age- and sex-matched healthy children were used as controls. They were referred to our outpatient clinic with suspicion of voiding disorders that were subsequently not confirmed.

To evaluate the designed laboratory parameters, the midstream first-morning urine and serum samples were simultaneously collected from each study participant. 
TABLE 2: The results of serum concentrations of MMPs and TIMPs in study A, B, and C and control groups.

\begin{tabular}{|c|c|c|c|c|c|}
\hline Variable & Group & $N$ & Median & Range & Statistical analysis \\
\hline \multirow{4}{*}{ MMP-1 (pg/ml) } & $\mathrm{A}$ & 25 & 297.5 & $410.0-1116.0$ & A vs. controls $p=0.3$ \\
\hline & $\mathrm{B}$ & 11 & 365.3 & $800.0-604.5$ & B vs. controls $p=0.2$ \\
\hline & $\mathrm{C}$ & 9 & 440.1 & $150.0-930.0$ & C vs. controls $p=0.3$ \\
\hline & Controls & 21 & 326.1 & $209.1-607.1$ & \\
\hline \multirow{4}{*}{ MMP-2 (pg/ml) } & $\mathrm{A}$ & 25 & $416.3 \times 10^{3}$ & $216.2 \times 10^{3}-674.5 \times 10^{3}$ & A vs. controls $p=0.06$ \\
\hline & $\mathrm{B}$ & 11 & $435.9 \times 10^{3}$ & $313.0 \times 10^{3}-635.6 \times 10^{3}$ & B vs. controls $p=0.6$ \\
\hline & $\mathrm{C}$ & 9 & $416.2 \times 10^{3}$ & $341.0 \times 10^{3}-569.7 \times 10^{3}$ & C vs. controls $p=0.23$ \\
\hline & Controls & 21 & $374.6 \times 10^{3}$ & $307.2 \times 10^{3}-726.2 \times 10^{3}$ & \\
\hline \multirow{4}{*}{ MMP-9 (pg/ml) } & A & 25 & $59.1 \times 10^{3}$ & $22.7 \times 10^{3}-159.8 \times 10^{3}$ & A vs. controls $p=0.0001$ \\
\hline & $\mathrm{B}$ & 11 & $54.2 \times 10^{3}$ & $25.1 \times 10^{3}-79.3 \times 10^{3}$ & B vs. controls $p=0.0002$ \\
\hline & $\mathrm{C}$ & 9 & $52.5 \times 10^{3}$ & $10.8 \times 10^{3}-94.7 \times 10^{3}$ & $\mathrm{C}$ vs. controls $p=0.001$ \\
\hline & Controls & 21 & $1.51 \times 10^{3}$ & $0.90 \times 10^{3}-2.2 \times 10^{3}$ & \\
\hline \multirow{4}{*}{ TIMP-1 (pg/ml) } & A & 25 & $71.7 \times 10^{3}$ & $8.20 \times 10^{3}-99.4 \times 10^{3}$ & A vs. controls $p=0.0001$ \\
\hline & $\mathrm{B}$ & 11 & $83.0 \times 10^{3}$ & $19.5 \times 10^{3}-105.1 \times 10^{3}$ & B vs. controls $p=0.0008$ \\
\hline & $\mathrm{C}$ & 9 & $75.7 \times 10^{3}$ & $14.4 \times 10^{3}-98.2 \times 10^{3}$ & $\mathrm{C}$ vs. controls $p=0.001$ \\
\hline & Controls & 21 & $13.3 \times 10^{3}$ & $6.50 \times 10^{3}-18.3 \times 10^{3}$ & \\
\hline \multirow{4}{*}{ TIMP-2 (pg/ml) } & $\mathrm{A}$ & 25 & $101.5 \times 10^{3}$ & $7.20 \times 10^{3}-120.6 \times 10^{3}$ & A vs. controls $p=0.04$ \\
\hline & $\mathrm{B}$ & 11 & $100.4 \times 10^{3}$ & $3.86 \times 10^{3}-122.4 \times 10^{3}$ & B vs. controls $p=0.04$ \\
\hline & $\mathrm{C}$ & 9 & $100.1 \times 10^{3}$ & $3.15 \times 10^{3}-120.1 \times 10^{3}$ & C vs. controls $p=0.04$ \\
\hline & Controls & 21 & $90.7 \times 10^{3}$ & $24.5 \times 10^{3}-173.5 \times 10^{3}$ & \\
\hline \multirow{4}{*}{ MMP-1/TIMP-1 } & A & 25 & 0.13 & $0.02-0.2$ & A vs. controls $p=0.5$ \\
\hline & $\mathrm{B}$ & 11 & 0.14 & $0.02-0.26$ & B vs. controls $p=0.3$ \\
\hline & C & 9 & 0.13 & $0.04-0.29$ & $\mathrm{C}$ vs. controls $p=0.3$ \\
\hline & Controls & 21 & 0.1 & $0.03-0.35$ & \\
\hline \multirow{4}{*}{ MMP-2/TIMP-1 } & $\mathrm{A}$ & 25 & 1.6 & $0.6-2.9$ & A vs. controls $p=0.01$ \\
\hline & $\mathrm{B}$ & 11 & 1.7 & $1.1-3.1$ & B vs. controls $p=0.002$ \\
\hline & C & 9 & 1.6 & $1.2-2.0$ & C vs. controls $p=0.001$ \\
\hline & Controls & 21 & 2.9 & $2.4-3.4$ & \\
\hline \multirow{4}{*}{ MMP-9/TIMP-1 } & A & 25 & 0.03 & $0.01-0.1$ & A vs. controls $p=0.03$ \\
\hline & $\mathrm{B}$ & 11 & 0.04 & $0.01-0.1$ & B vs. controls $p=0.04$ \\
\hline & C & 9 & 0.15 & $0.1-2.1$ & $C$ vs. controls $p=0.04$ \\
\hline & Controls & 21 & 0.1 & $0.1-0.2$ & \\
\hline \multirow{4}{*}{ MMP-1/TIMP-2 } & A & 25 & 0.09 & $0.04-0.2$ & A vs. controls $p=0.4$ \\
\hline & $\mathrm{B}$ & 11 & 0.05 & $0.01-0.2$ & B vs. controls $p=0.1$ \\
\hline & C & 9 & 0.07 & $0.03-0.14$ & $\mathrm{C}$ vs. controls $p=0.4$ \\
\hline & Controls & 21 & 0.06 & $0.02-0.1$ & \\
\hline \multirow{4}{*}{ MMP-2/TIMP-2 } & A & 25 & 3.2 & $0.6-5.0$ & A vs. controls $p=0.88$ \\
\hline & $\mathrm{B}$ & 11 & 3.4 & $2.8-5.3$ & B vs. controls $p=0.58$ \\
\hline & $\mathrm{C}$ & 9 & 3.0 & $1.9-4.8$ & $\mathrm{C}$ vs. controls $p=0.09$ \\
\hline & Controls & 21 & 3.3 & $2.0-5.0$ & \\
\hline
\end{tabular}


TABLE 2: Continued.

\begin{tabular}{lccccc}
\hline Variable & Group & $N$ & Median & Range & Statistical analysis \\
\hline & A & 25 & 0.02 & $0.01-0.3$ & A vs. controls $p=0.04$ \\
MMP-9/TIMP-2 & B & 11 & 0.03 & $0.01-0.3$ & B vs. controls $p=0.04$ \\
& C & 9 & 0.1 & $0.07-0.3$ & C vs. controls $p=0.1$ \\
& Controls & 21 & 0.09 & $0.03-0.28$ & \\
\hline
\end{tabular}

MMP-1, MMP-2, and MMP-9 and TIMP-1 and TIMP-2 concentrations were measured using specific enzyme-linked immunosorbent assay (ELISA) kits following the manufacturer's instructions (R\&D Systems).

Serum and urinary creatinine concentrations were determined by Jaffe's test. Standard laboratory techniques were used to assess the magnitude of proteinuria.

Urinary excretions of MMP-1, MMP-2, MMP-3, TIMP-1, TIMP-2, and protein were expressed as ratios over creatinine concentration ( $\mathrm{pg} / \mathrm{mg} \mathrm{Cr}$ and $\mathrm{mg} / \mathrm{mg} \mathrm{Cr}$, respectively).

In addition, to evaluate the ECM remodelling process activity, the serum and urinary MMP-1, -2, -9/TIMP-1, -2 ratios (pg/pg) were also calculated.

The statistical analysis was performed using STATISTICA 12.5. The differences between the groups were assessed using a nonparametric Mann-Whitney $U$ test, while correlation coefficients were calculated using the Spearman test. A $p$ value $\leq 0.05$ was considered significant. To determinate the diagnostic utility of the evaluated biomarkers, the receiver operating characteristic (ROC) curves were analysed.

\section{Ethics Statement}

The study was approved by the ethics committee of the Medical University of Lublin.

Informed consent was obtained from all parents or legal representatives, as well as, according to Polish regulations from patients $>16$ years of age.

\section{Results}

Tables 2 and 3 show the results of the assessed biomarkers in the study groups compared to the controls. In group A, median serum concentrations of MMP-9, TIMP-1, TIMP-2, and median urinary MMP-2, $-9 / \mathrm{Cr}$ and TIMP $-1,-2 / \mathrm{Cr}$ ratios were significantly higher than those in the controls $(p<0.05)$. In comparison with the controls, group A showed significantly lower median values of serum MMP-2/TIMP-1, MMP-9/TIMP-1, -2, and urinary MMP-2/TIMP-1, -2 ratios $(p<0.05)$. In comparison with the controls, patients from group B showed significantly higher median serum MMP-9, TIMP-1, TIMP-2 concentrations, lower median serum MMP-2/TIMP-1, MMP-9/TIMP-1, -2 ratios, and higher median urinary MMP-9/Cr and TIMP-2/Cr ratios $(p<0.05)$. In group $C$, significantly higher median serum MMP-9, TIMP-1, TIMP-2 concentrations, lower median serum MMP-2, -9/TIMP-1 ratios, and higher median urinary MMP-9/Cr and TIMP-2/Cr ratios in comparison with the controls were observed $(p<0.05)$.
Patients with ON had significantly higher median serum MMP-9 concentration (Figure 1) and significantly lower median serum MMP-9/TIMP-1, -2 ratios in comparison with those without this diagnosis $(p=0.03, p=0.01$, and $p=0.002$, respectively). In patients with nonglomerular proteinuria, a significantly higher median serum TIMP-1 concentration, a higher median urinary TIMP-2/Cr ratio, and a significantly lower serum MMP-9/TIMP-1 ratio compared to those without this symptom were revealed $(p<0.05)$ (Figure 2). No correlations between the measured biomarkers and the relative function of the obstructed kidney were observed.

The ROC curve analysis showed a promising diagnostic profile for the detection of ON for serum MMP-9 (area under the curve (AUC) of 0.722 , optimal cut-off value of $526200 \mathrm{pg} / \mathrm{ml}$ with a sensitivity and specificity of 71 and $66.7 \%$, respectively) and also for serum MMP-9/TIMP-1 and MMP-9/TIMP-2 ratios (AUCs of 0.758 and 0.697, optimal cut-off values of 2.961 and $3.838 \mathrm{pg} / \mathrm{pg}$ with sensitivities of 58.1 and $83.9 \%$ and specificities of 88.9 and $55.6 \%$, respectively) (Figure 3).

\section{Discussion}

Chronic HN may lead to the development of progressive kidney fibrosis determined as $\mathrm{ON}$ and the impairment of renal function [29]. Therefore, identification of biomarkers of tubulointerstitial injury would be useful in therapeutic decisions and evaluation of ON progression. Among potential candidates are selected MMPs and TIMPs which contribute to the fibrotic process within the renal tissue [7, 30-32]. However, the amount of relevant clinical data is limited.

The role of MMP-2 in renal fibrosis was shown in several experimental studies [11, 18, 19]. Accordingly, rabbits with experimentally induced UPJO showed higher expression of MMP-2 in the renal cortex [33]. Furthermore, Tveitarås et al. [34] found that MMP-2 knockout and heterozygote mice are protected from kidney fibrosis despite the presence of coexisting UPJO. In a few clinical studies, increased urinary MMP-2 excretion was observed at an early stage of chronic nephropathy [35-38]. Other studies reported patients with CKD with associated progressive kidney fibrosis accompanied by increased serum MMP-2 concentrations $[21,39-44]$. In addition, increased serum MMP-2 levels were observed in patients with interstitial fibrosis/tubular atrophy in renal allograft [38]. Our study confirmed an increased release of MMP-2 in chronic uropathy. Patients with HN grades three and four were characterised by higher concentrations of urinary MMP-2 and lower serum and urinary MMP-2/TIMP-1, -2 ratios in comparison with the controls. 
TABLE 3: The results of urinary excretions of MMPs and TIMPs in study groups A, B, and C and control groups.

\begin{tabular}{|c|c|c|c|c|c|}
\hline Variable & Group & $N$ & Median & Range & Statistical analysis \\
\hline \multirow{4}{*}{ MMP-1/Cr (pg/mg) } & $\mathrm{A}$ & 25 & 171.2 & $89.5-1125.1$ & A vs. controls $p=0.3$ \\
\hline & $\mathrm{B}$ & 11 & 170.2 & $93.4-1521.5$ & B vs. controls $p=0.4$ \\
\hline & $\mathrm{C}$ & 9 & 169.8 & $47.6-765.4$ & $C$ vs. controls $p=0.4$ \\
\hline & Controls & 21 & 164.4 & 86.9-1488.8 & \\
\hline \multirow{4}{*}{ MMP-2/Cr (pg/mg) } & A & 25 & 401.2 & $120.2-2310.0$ & A vs. controls $p=0.14$ \\
\hline & $\mathrm{B}$ & 11 & 323.1 & 87.9-628.3 & B vs. controls $p=0.6$ \\
\hline & $\mathrm{C}$ & 9 & 315.8 & 121.1-399.8 & $\mathrm{C}$ vs. controls $p=0.24$ \\
\hline & Controls & 21 & 305.9 & $129.3-979.3$ & \\
\hline \multirow{4}{*}{ MMP-9/Cr (pg/mg) } & A & 25 & $1.87 \times 10^{3}$ & $0.32 \times 10^{3}-7.13 \times 10^{3}$ & A vs. controls $p=0.002$ \\
\hline & B & 11 & $1.01 \times 10^{3}$ & $0.22 \times 10^{3}-9.36 \times 10^{3}$ & B vs. controls $p=0.009$ \\
\hline & $\mathrm{C}$ & 9 & $2.68 \times 10^{3}$ & $0.56 \times 10^{3}-3.67 \times 10^{3}$ & $\mathrm{C}$ vs. controls $p=0.003$ \\
\hline & Controls & 21 & $0.49 \times 10^{3}$ & $0.14 \times 10^{3}-1.52 \times 10^{3}$ & \\
\hline \multirow{4}{*}{ TIMP-1/Cr (pg/mg) } & A & 25 & $7.13 \times 10^{3}$ & $4.84 \times 10^{3}-17.9 \times 10^{3}$ & A vs. controls $p=0.04$ \\
\hline & B & 11 & $4.98 \times 10^{3}$ & $1.23 \times 10^{3}-8.77 \times 10^{3}$ & B vs. controls $p=0.36$ \\
\hline & $\mathrm{C}$ & 9 & $4.44 \times 10^{3}$ & $2.56 \times 10^{3}-7.08 \times 10^{3}$ & $\mathrm{C}$ vs. controls $p=0.88$ \\
\hline & Controls & 21 & $5.42 \times 10^{3}$ & $2.05 \times 10^{3}-13.7 \times 10^{3}$ & \\
\hline \multirow{4}{*}{ TIMP-2/Cr (pg/mg) } & $\mathrm{A}$ & 25 & $2.98 \times 10^{3}$ & $0.12 \times 10^{3}-6.69 \times 10^{3}$ & A vs. controls $p=0.04$ \\
\hline & $\mathrm{B}$ & 11 & $2.85 \times 10^{3}$ & $0.34 \times 10^{3}-9.26 \times 10^{3}$ & B vs. controls $p=0.02$ \\
\hline & $\mathrm{C}$ & 9 & $2.19 \times 10^{3}$ & $1.40 \times 10^{3}-10.6 \times 10^{3}$ & $\mathrm{C}$ vs. controls $p=0.01$ \\
\hline & Controls & 21 & $0.43 \times 10^{3}$ & $0.10 \times 10^{3}-2.67 \times 10^{3}$ & \\
\hline \multirow{4}{*}{ MMP-1/TIMP-1 } & $\mathrm{A}$ & 25 & 0.38 & $0.11-1.0$ & A vs. controls $p=0.2$ \\
\hline & $\mathrm{B}$ & 11 & 0.32 & $0.11-0.54$ & B vs. controls $p=0.06$ \\
\hline & C & 9 & 0.5 & $0.30-0.71$ & C vs. controls $p=0.7$ \\
\hline & Controls & 21 & 0.44 & $0.11-0.66$ & \\
\hline \multirow{4}{*}{ MMP-2/TIMP-1 } & A & 25 & 0.1 & $0.04-1.0$ & A vs. controls $p=0.001$ \\
\hline & $\mathrm{B}$ & 11 & 0.7 & $0.11-2.4$ & B vs. controls $p=0.1$ \\
\hline & C & 9 & 0.8 & $0.11-0.92$ & $\mathrm{C}$ vs. controls $p=0.2$ \\
\hline & Controls & 21 & 0.9 & $0.20-4.2$ & \\
\hline \multirow{4}{*}{ MMP-9/TIMP-1 } & A & 25 & 1.2 & $0.03-1.51$ & A vs. controls $p=0.87$ \\
\hline & B & 11 & 1.9 & $0.1-6.4$ & B vs. controls $p=0.7$ \\
\hline & C & 9 & 0.9 & $0.1-1.7$ & $\mathrm{C}$ vs. controls $p=0.09$ \\
\hline & Controls & 21 & 1.2 & $0.35-16.2$ & \\
\hline \multirow{4}{*}{ MMP-1/TIMP-2 } & $\mathrm{A}$ & 25 & 0.2 & $0.1-0.3$ & A vs. controls $p=0.8$ \\
\hline & $\mathrm{B}$ & 11 & 0.12 & 0.03-0.4 & B vs. controls $p=0.1$ \\
\hline & C & 9 & 0.1 & $0.03-0.23$ & $\mathrm{C}$ vs. controls $p=0.8$ \\
\hline & Controls & 21 & 0.1 & $0.02-0.2$ & \\
\hline \multirow{4}{*}{ MMP-2/TIMP-2 } & $\mathrm{A}$ & 25 & 0.05 & $0.02-0.28$ & A vs. controls $p=0.001$ \\
\hline & $\mathrm{B}$ & 11 & 0.11 & $0.01-0.3$ & B vs. controls $p=0.6$ \\
\hline & C & 9 & 0.09 & $0.02-0.41$ & $\mathrm{C}$ vs. controls $p=0.1$ \\
\hline & Controls & 21 & 0.1 & $0.02-0.31$ & \\
\hline
\end{tabular}


TABLE 3: Continued.

\begin{tabular}{lccccc}
\hline Variable & Group & $N$ & Median & Range & Statistical analysis \\
\hline & A & 25 & 0.18 & $0.06-0.28$ & A vs. controls $p=0.57$ \\
MMP-9/TIMP-2 & B & 11 & 0.2 & $0.09-0.28$ & B vs. controls $p=0.7$ \\
& C & 9 & 0.09 & $0.03-0.2$ & C vs. controls $p=0.39$ \\
& Controls & 21 & 0.1 & $0.04-0.32$ & \\
\hline
\end{tabular}

Cr: creatinine.

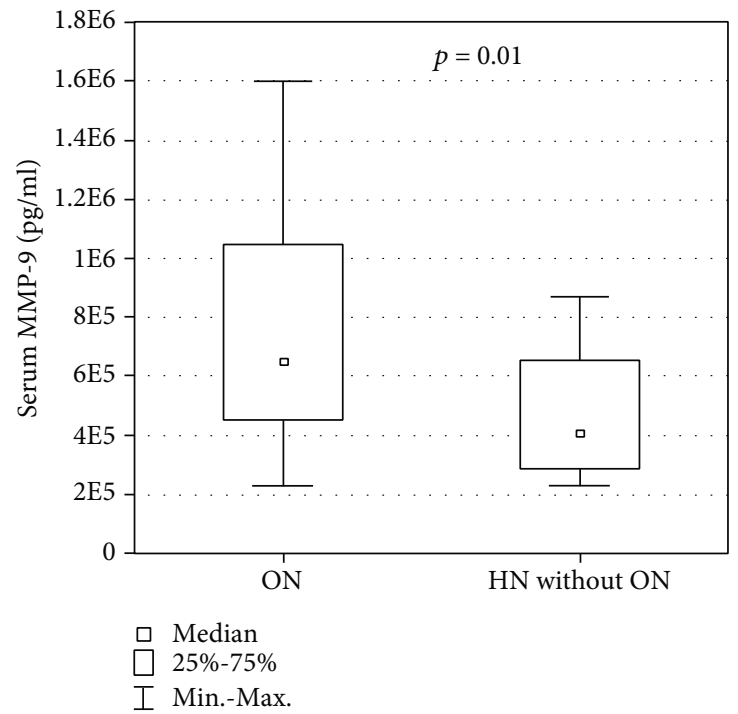

(a)

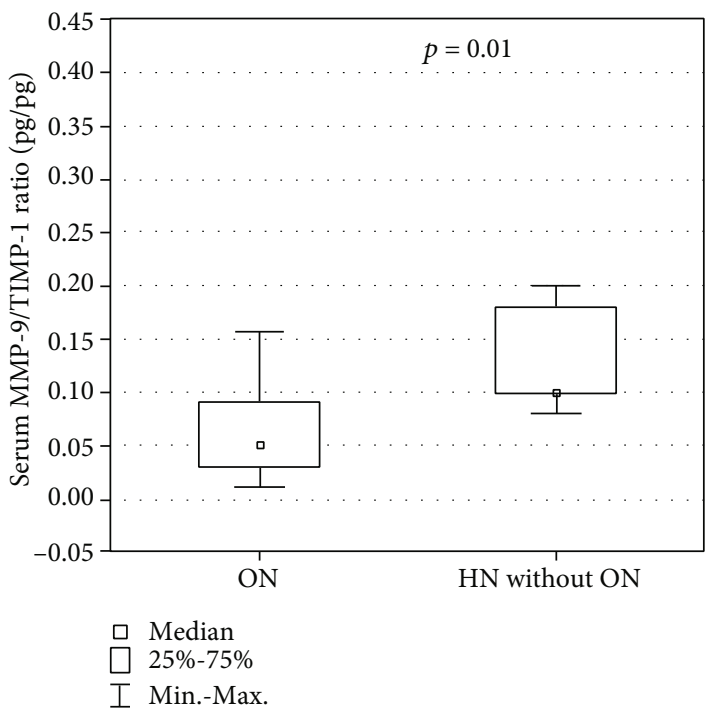

(b)

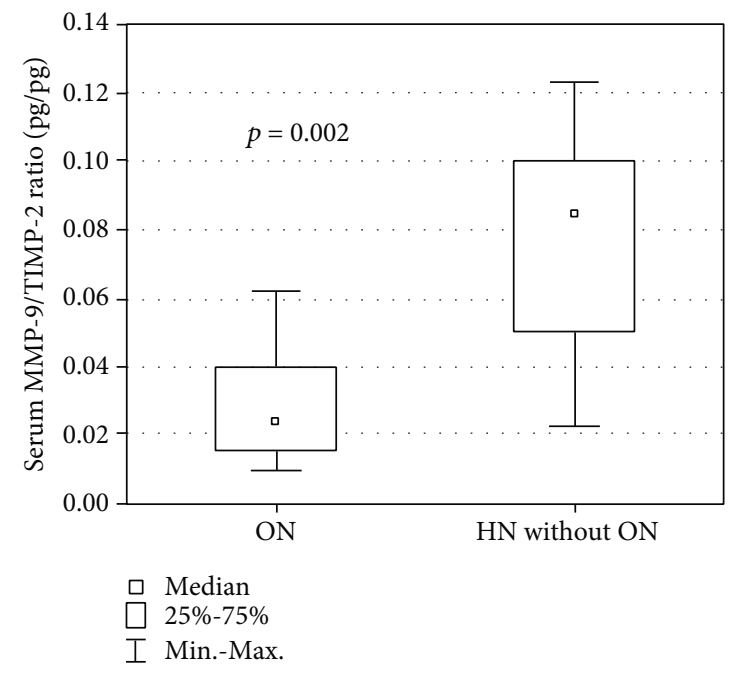

(c)

FIgURE 1: Serum concentration of (a) MMP-9 and serum ratios of (b) MMP-9/TIMP-1 and (c) MMP-9/TIMP-2 in patients with obstructive nephropathy $(\mathrm{ON})$ and hydronephrosis without obstructive nephropathy (HN without $\mathrm{ON})$.

The latter was also found in patients with mild stages of HN. These results may indicate an association of HN severity, accumulation of ECM in the renal parenchyma, and the development of tubulointerstitial fibrosis. It suggests that serum and urinary MMP-2 could serve as clinical markers of ON. The contribution of MMP-9 in renal fibrosis was evaluated more extensively than MMP-2. Several reports showed increased serum and urinary MMP-9 activity in patients with CKD [40-42]. Abedi and Mohammadjafari [45] postulated that urinary MMP-9 and TIMP-1 may be markers of renal scarring in children with urinary tract infections. Other authors [46] suggested the usefulness of the measurement of urinary MMP-9 excretion and the urinary MMP9/TIMP-1 ratio for the prediction of vesicoureteral reflux in 


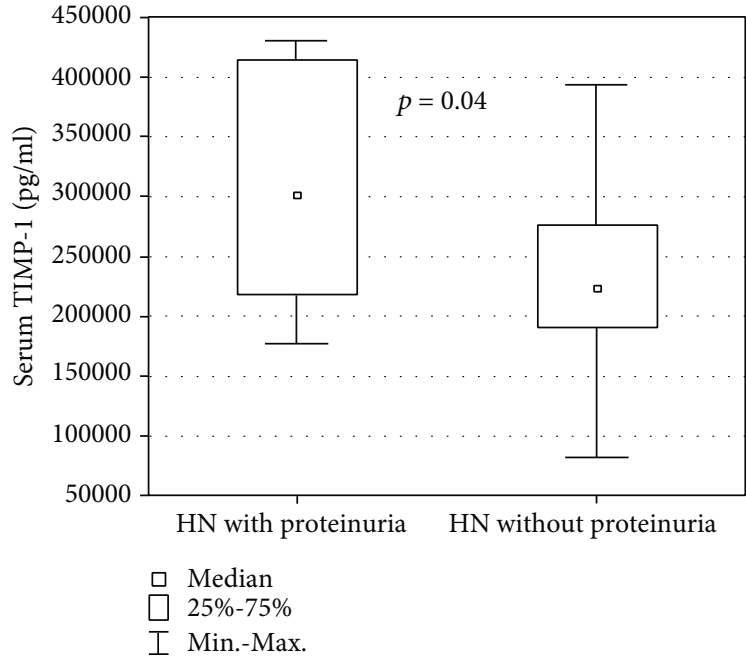

(a)

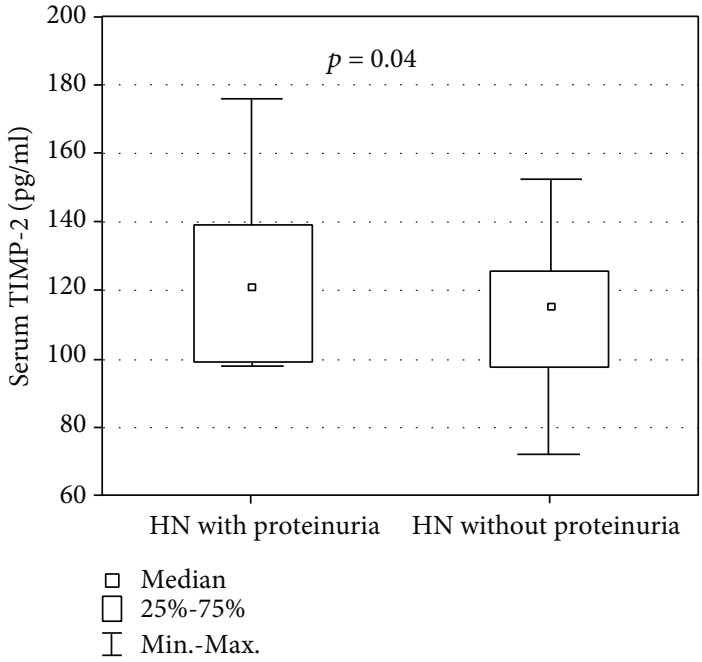

(b)

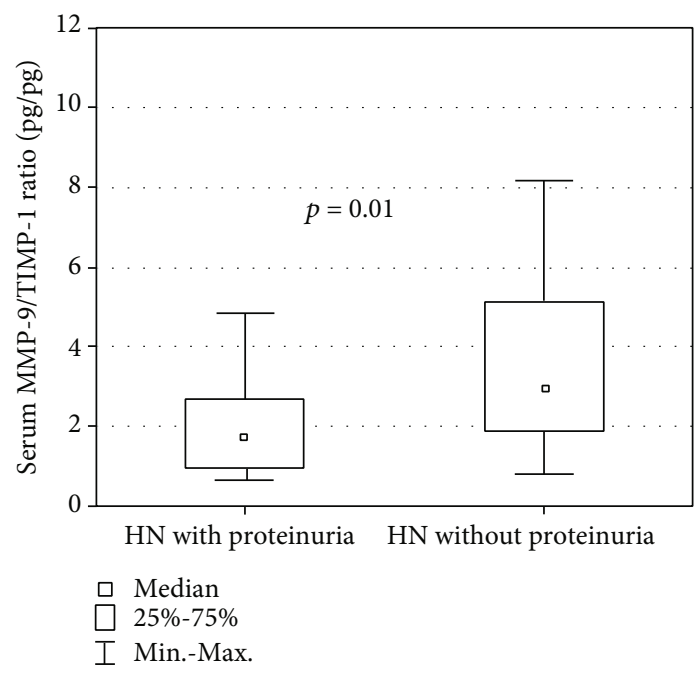

(c)

FIgURE 2: Serum concentrations of (a) TIMP-1 and (b) TIMP-2 and (c) serum MMP-9/TIMP-1 ratio in hydronephrotic patients with and without proteinuria.

neonates with antenatal HN. Tian et al. [47] reported that urinary MMP-9 and TIMP-1 may be noninvasive biomarkers in children with UPJO. On the contrary, the data published by Reis et al. [48] showed that higher expression of MMP-9 is a marker for a good surgical outcome in children with UPJO. The authors believe that activation of MMP-9 can reflect increased degradation processes in the ECM. The results of our study suggest increased synthesis and release of MMP-9 in patients with UPJO and the development of renal fibrosis. Children with $\mathrm{HN}$ were characterised by significantly increased serum and urinary concentrations of MMP-9. Patients with HN grades two, three, and four also had lower serum MMP-9/TIMP-1, -2 ratios in comparison with the controls. Moreover, in patients with ON, increased serum MMP-9 concentration and decreased serum MMP-9/TIMP-1, -2 ratios were observed. In addition, decreased serum MMP-9/TIMP-1 ratio was also found in patients with $\mathrm{HN}$ and nonglomerular proteinuria.
Finally, the promising diagnostic profiles of serum MMP-9 and serum MMP-9/TIMP-1, -2 ratios for the detection of ON were confirmed in the ROC curve analysis.

There is only a little data concerning the role of MMP-1 in renal fibrosis. Hirt-Minkowski et al. [49] revealed a significant positive correlation between serum and urinary concentrations of MMP-1 and TIMP-1 and advanced or even initial interstitial fibrosis in renal allograft. In contrast, the data in this study did not show differences in serum and urinary MMP-1 between the groups of patients with $\mathrm{HN}$, with or without $\mathrm{ON}$, and the controls. This may suggest different pathways of fibrosis between native and transplanted kidneys. Therefore, MMP-1 seems not to be a suitable biomarker of CKD caused by ON.

Some experimental studies found higher expression of TIMPs, particularly TIMP-1, in the renal tissue in the course of various kidney diseases $[32,50]$. This was confirmed in patients with CKD of different aetiology [23, 51]. It was 


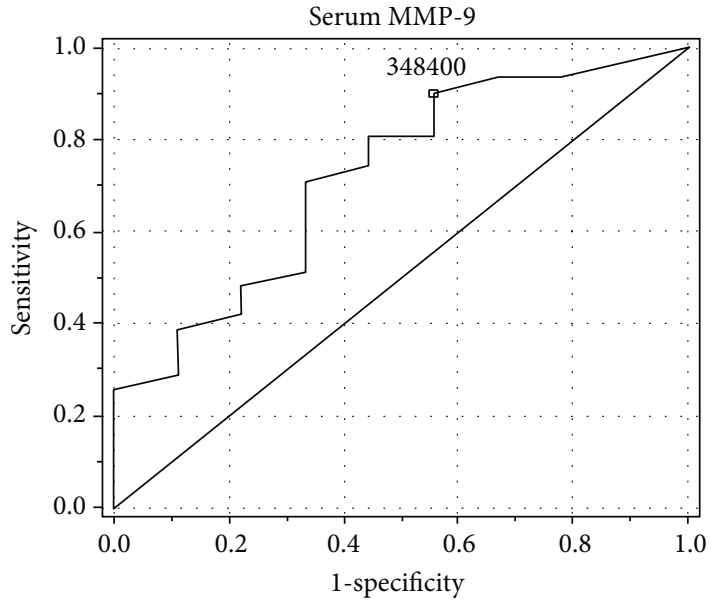

(a)

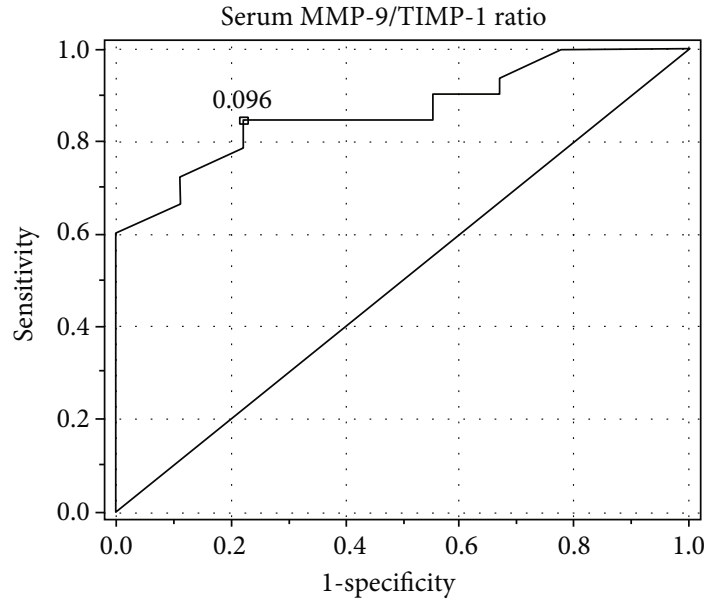

(b)

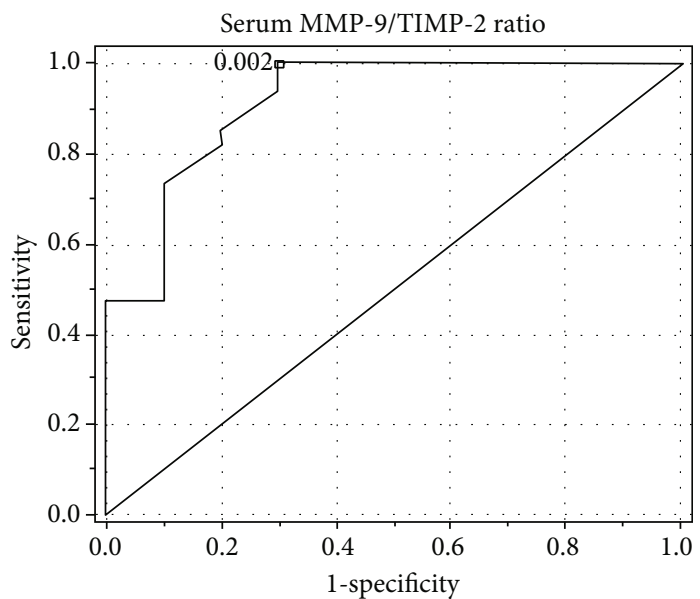

(c)

FIgURE 3: ROC analysis for the (a) serum MMP-9, (b) serum MMP-9/TIMP-1, and (a) serum MMP-9/TIMP-2 ratios in the detection of obstructive nephropathy (patients with obstructive nephropathy $(\mathrm{ON})$ vs. patients with hydronephrosis without obstructive nephropathy $(\mathrm{HN}$ without $\mathrm{ON})$ ).

found that increased urinary excretion of TIMP-1 was observed particularly in patients with UPJO [47] and vesicoureteral reflux [46]. This parameter was also proposed as a predictor of renal scarring [45]. In our study, patients with HN showed higher serum concentrations of TIMP-1 and TIMP-2 and urinary excretions of TIMP-2 in comparison with the controls. Significantly higher excretions of urinary TIMP-1 were found only in children with HN grades three and four. Moreover, in patients with nonglomerular proteinuria, significantly higher serum TIMP-1, higher urinary TIMP-2, and lower serum MMP-9/TIMP-1 ratio compared to those without this symptom were detected. This may confirm the increased ECM remodelling inhibition processes in patients with $\mathrm{HN}$ and the higher risk of the development of tubulointerstitial fibrosis.

This study showed that in patients with $\mathrm{HN}$, independent of the grade, the process of renal tubulointerstitial fibrosis is activated. The development or inhibition of this condition is probably dependent on multiple factors, such as the progression of hydronephrosis, urinary tract infections, and also individual considerations. Monitoring of urinary and serum MMP-2, -9 and MMP-2, -9/TIMP-1, -2 ratios may become useful in determining the progression of renal fibrosis and better stratification of patients with $\mathrm{ON}$.

The main limitation of this preliminary study is a relatively small number of patients. Further investigations in patients with $\mathrm{HN}$ are required to confirm the utility of serum and urinary MMPs and TIMPs in the diagnosis of ON.

\section{Conclusions}

In conclusion, the results of our study suggest that patients with $\mathrm{HN}$, particularly grades three and four, are at higher risk of renal tubulointerstitial fibrosis. Urinary $\mathrm{MMP}-2 / \mathrm{Cr}$ and MMP-9/Cr, serum MMP-9, and serum and urinary MMP-2, -9/TIMP-1, -2 may be considered as noninvasive markers of this condition. Additionally, serum MMP-9 and MMP-9/TIMP-1, -2 may become promising markers of $\mathrm{ON}$. Therefore, the assessment of these parameters could be useful for follow-up of patients with HN caused by UPJO. 


\section{Data Availability}

The data used to support the findings of this study are available from the corresponding author upon request.

\section{Conflicts of Interest}

The authors declare that there is no conflict of interest regarding the publication of this paper.

\section{References}

[1] R. L. Chevalier, M. S. Forbes, and B. A. Thornhill, "Ureteral obstruction as a model of renal interstitial fibrosis and obstructive nephropathy," Kidney International, vol. 75 , no. 11, pp. 1145-1152, 2009.

[2] R. L. Chevalier, B. A. Thornhill, M. S. Forbes, and S. C. Kiley, "Mechanisms of renal injury and progression of renal disease in congenital obstructive nephropathy," Pediatric Nephrology, vol. 25, no. 4, pp. 687-697, 2010.

[3] M. Toi, S. Ishigaki, and T. Tominaga, "Metalloproteinases and tissue inhibitors of metalloproteinases," Breast Cancer Research and Treatment, vol. 52, no. 1-3, pp. 113-124, 1998.

[4] R. Visse and H. Nagase, "Matrix metalloproteinases and tissue inhibitors of metalloproteinases," Circulation Research, vol. 92, no. 8, pp. 827-839, 2003.

[5] J. M. Catania, G. Chen, and A. R. Parrish, "Role of matrix metalloproteinases in renal pathophysiologies," American Journal of Physiology-Renal Physiology, vol. 292, no. 3, pp. F905-F911, 2007.

[6] H. Nagase, R. Visse, and G. Murphy, "Structure and function of matrix metalloproteinases and TIMPs," Cardiovascular Research, vol. 69, no. 3, pp. 562-573, 2006.

[7] A. A. Eddy, "Molecular basis of renal fibrosis," Pediatric Nephrology, vol. 15, no. 3-4, pp. 290-301, 2000.

[8] T. K. Tan, G. Zheng, T. T. Hsu et al., "Macrophage matrix metalloproteinase- 9 mediates epithelial-mesenchymal transition in vitro in murine renal tubular cells," The American Journal of Pathology, vol. 176, no. 3, pp. 1256-1270, 2010.

[9] L. Aresu, S. Benali, S. Garbisa, E. Gallo, and M. Castagnaro, "Matrix metalloproteinases and their role in the renal epithelial mesenchymal transition," Histology and Histopathology, vol. 26, no. 3, pp. 307-313, 2011.

[10] Y. Okada, Y. Gonoji, K. Naka et al., "Matrix metalloproteinase 9 (92-kDa gelatinase/type IV collagenase) from HT 1080 human fibrosarcoma cells: purification and activation of the precursor and enzymic properties," The Journal of Biological Chemistry, vol. 267, no. 30, pp. 21712-21719, 1992.

[11] M. W. Olson, M. Toth, D. C. Gervasi, Y. Sado, Y. Ninomiya, and R. Fridman, "High affinity binding of latent matrix metalloproteinase-9 to the alpha2 (IV) chain of collagen IV," The Journal of Biological Chemistry, vol. 273, no. 17, pp. 10672-10681, 1998.

[12] L. Schaefer, X. Han, N. Gertz et al., "Tubular gelatinase A (MMP-2) and its tissue inhibitors in polycystic kidney disease in the Han:SPRD rat," Kidney International, vol. 49, no. 1, pp. 75-81, 1996.

[13] K. A. Inkinen, A. P. Soots, L. A. Krogerus, I. T. Lautenschlager, and J. P. Ahonen, "Fibrosis and matrix metalloproteinases in rat renal allografts," Transplant International, vol. 18, no. 5, pp. 506-512, 2005.
[14] M. Tomita, H. Koike, G. D. Han, F. Shimizu, and H. Kawachi, "Decreased collagen-degrading activity could be a marker of prolonged mesangial matrix expansion," Clinical and Experimental Nephrology, vol. 8, no. 1, pp. 17-26, 2004.

[15] R. Piedagnel, G. Murphy, P. M. Ronco, and B. Lelongt, "Matrix metalloproteinase 2(MMP2) and MMP9 are produced by kidney collecting duct principal cells but are differentially regulated by SV40 large-T, arginine vasopressin, and epidermal growth factor," The Journal of Biological Chemistry, vol. 274, no. 3, pp. 1614-1620, 1999.

[16] T. Kuroda, Y. Yoshida, J. Kamiie et al., "Expression of MMP-9 in mesangial cells and its changes in anti-GBM glomerulonephritis in WKY rats," Clinical and Experimental Nephrology, vol. 8, no. 3, pp. 206-215, 2004.

[17] K. U. Ogbureke and L. W. Fisher, "Renal expression of SIBLING proteins and their partner matrix metalloproteinases (MMPs)," Kidney International, vol. 68, no. 1, pp. 155-166, 2005.

[18] Q. Li, P. W. Park, C. L. Wilson, and W. C. Parks, "Matrilysin shedding of syndecan-1 regulates chemokine mobilization and transepithelial efflux of neutrophils in acute lung injury," Cell, vol. 111, no. 5, pp. 635-646, 2002.

[19] M. Toth, I. Chvyrkova, M. M. Bernardo, S. Hernandez-Barrantes, and R. Fridman, "Pro-MMP-9 activation by the MT1MMP/MMP-2 axis and MMP-3: role of TIMP-2 and plasma membranes," Biochemical and Biophysical Research Communications, vol. 308, no. 2, pp. 386-395, 2003.

[20] T. K. Tan, G. Zheng, T.-T. Hsu et al., "Matrix metalloproteinase- 9 of tubular and macrophage origin contributes to the pathogenesis of renal fibrosis via macrophage recruitment through osteopontin cleavage," Laboratory Investigation, vol. 93, no. 4, pp. 434-449, 2013.

[21] K. Pawlak, M. Mysliwiec, and D. Pawlak, "Peripheral blood level alterations of MMP-2 and MMP-9 in patients with chronic kidney disease on conservative treatment and on hemodialysis," Clinical Biochemistry, vol. 44, no. 10-11, pp. 838-843, 2011.

[22] H. P. Marti, "Role of matrix metalloproteinases in the progression of renal lesions," Presse Médicale, vol. 29, pp. 811-817, 2000.

[23] K. Musiał, A. Bargenda, and D. Zwolińska, "Urine survivin, Ecadherin and matrix metalloproteinases as novel biomarkers in children with chronic kidney disease," Biomarkers, vol. 20, no. 3, pp. 177-182, 2015.

[24] C. J. Morrison, G. S. Butler, D. Rodríguez, and C. M. Overall, "Matrix metalloproteinase proteomics: substrates, targets, and therapy," Current Opinion in Cell Biology, vol. 21, no. 5, pp. 645-653, 2009.

[25] C. M. Overall, "Molecular determinants of metalloproteinase substrate specificity: matrix metalloproteinase substrate binding domains, modules, and exosites," Molecular Biotechnology, vol. 22, no. 1, pp. 051-086, 2002.

[26] K. Musiał and D. Zwolińska, "Matrix metalloproteinases (MMP-2, 9) and their tissue inhibitors (TIMP-1,2) as novel markers of stress response and atherogenesis in children with chronic kidney disease (CKD) on conservative treatment," Cell Stress \& Chaperones, vol. 16, no. 1, pp. 97-103, 2011.

[27] A. Onen, "An alternative grading system to refine the criteria for severity of hydronephrosis and optimal treatment guidelines in neonates with primary UPJ-type hydronephrosis," Journal of Pediatric Urology, vol. 3, no. 3, pp. 200-205, 2007. 
[28] G. J. Schwartz, L. P. Brion, and A. Spitzer, "The use of plasma creatinine concentration for estimating glomerular filtration rate in infants, children, and adolescents," Pediatric Clinics of North America, vol. 34, no. 3, pp. 571-590, 1987.

[29] R. L. Chevalier, "Congenital urinary tract obstruction: the long view," Advances in Chronic Kidney Disease, vol. 22, no. 4, pp. 312-319, 2015.

[30] G. Qing-Hua, L. Ju-Ming, P. Chang-Yu, L. Zhao-Hui, Z. Xiao-Man, and M. Yi-Ming, "The kidney expression of matrix metalloproteinase-9 in the diabetic nephropathy of Kkay mice," Journal of Diabetes and its Complications, vol. 22, no. 6, pp. 408-412, 2008.

[31] A. K. Ahmed, J. L. Haylor, A. M. el Nahas, and T. S. Johnson, "Localization of matrix metalloproteinases and their inhibitors in experimental progressive kidney scarring," Kidney International, vol. 71, no. 8, pp. 755-763, 2007.

[32] S. Ishidoya, J. Morrissey, R. McCracken, and S. Klahr, "Delayed treatment with enalapril halts tubulointerstitial fibrosis in rats with obstructive nephropathy," Kidney International, vol. 49, no. 4, pp. 1110-1119, 1996.

[33] A. K. Sharma, S. M. Mauer, Y. Kim, and A. F. Michael, "Altered expression of matrix metalloproteinase-2, TIMP, and TIMP-2 in obstructive nephropathy," The Journal of Laboratory and Clinical Medicine, vol. 125, no. 6, pp. 754-761, 1995.

[34] M. K. Tveitarås, T. Skogstrand, S. Leh et al., "Matrix metalloproteinase- 2 knockout and heterozygote mice are protected from hydronephrosis and kidney fibrosis after unilateral ureteral obstruction," PLoS One, vol. 10, no. 12, 2015.

[35] P. Morillas, J. Quiles, H. de Andrade et al., "Circulating biomarkers of collagen metabolism in arterial hypertension: relevance of target organ damage," Journal of Hypertension, vol. 31, no. 8, pp. 1611-1617, 2013.

[36] N. Altemtam, M. E. Nahas, and T. Johnson, "Urinary matrix metalloproteinase activity in diabetic kidney disease: a potential marker of disease progression," Nephron Extra, vol. 2, no. 1, pp. 219-232, 2012.

[37] K. M. Thrailkill, R. C. Bunn, C. S. Moreau et al., "Matrix metalloproteinase-2 dysregulation in type 1 diabetes," Diabetes Care, vol. 30, no. 9, pp. 2321-2326, 2007.

[38] S. Rödder, A. Scherer, F. Raulf et al., "Renal allografts with IF/TA display distinct expression profiles of metzincins and related genes," American Journal of Transplantation, vol. 9, no. 3, pp. 517-526, 2009.

[39] K. Musiał and D. Zwolińska, "Novel indicators of fibrosisrelated complications in children with chronic kidney disease," Clinica Chimica Acta, vol. 430, pp. 15-19, 2014.

[40] K. Musiał and D. Zwolińska, "New markers of apoptosis in children on chronic dialysis," Apoptosis, vol. 18, no. 1, pp. 77-84, 2013.

[41] K. Musiał and D. Zwolińska, "Matrix metalloproteinases and soluble Fas/FasL system as novel regulators of apoptosis in children and young adults on chronic dialysis," Apoptosis, vol. 16, no. 7, pp. 653-659, 2011.

[42] H. R. Chang, S. F. Yang, M. L. Li, C. C. Lin, Y. S. Hsieh, and J. D. Lian, "Relationships between circulating matrix metalloproteinase- 2 and -9 and renal function in patients with chronic kidney disease," Clinica Chimica Acta, vol. 366, no. 1-2, pp. 243-248, 2006.

[43] S. Cheng, A. S. Pollock, R. Mahimkar et al., "Matrix metalloproteinase 2 and basement membrane integrity: a unifying mechanism for progressive renal injury," The FASEB Journal, vol. 20, no. 11, pp. 1898-1900, 2006.

[44] I. B. McKittrick, Y. Bogaert, K. Nadeau et al., "Urinary matrix metalloproteinase activities: biomarkers for plaque angiogenesis and nephropathy in diabetes," American Journal of Physiology-Renal Physiology, vol. 301, no. 6, pp. F1326F1333, 2011.

[45] S. M. Abedi and H. Mohammadjafari, "Urinary matrix metalloproteinase 9 and tissue inhibitor of metalloproteinase 1 biomarkers for predicting renal scar in children with urinary tract infection," Turkish Journal of Urology, vol. 43, no. 4, pp. 536-542, 2017.

[46] H. Mohammadjafari, A. Rafiei, M. Abedi, A. Aalaee, and E. Abedi, "The role of urinary TIMP1 and MMP9 levels in predicting vesicoureteral reflux in neonates with antenatal hydronephrosis," Pediatric Nephrology, vol. 29, no. 5, pp. 871-878, 2014.

[47] F. Tian, C. Gu, Z. Zhao, L. Li, S. Lu, and Z. Li, "Urinary Emmprin, matrix metalloproteinase 9 and tissue inhibitor of metalloproteinase 1 as potential biomarkers in children with ureteropelvic junction narrowing on conservative treatment," Nephrology (Carlton, Vic.), vol. 20, no. 3, pp. 194-200, 2015.

[48] S. T. Reis, K. R. M. Leite, N. I. Viana et al., "MMP9 overexpression is associated with good surgical outcome in children with UPJO: preliminary results," BMC Urology, vol. 16, no. 1, p. 44, 2016.

[49] P. Hirt-Minkowski, H. P. Marti, G. Hönger et al., "Correlation of serum and urinary matrix metalloproteases/tissue inhibitors of metalloproteases with subclinical allograft fibrosis in renal transplantation," Transplant Immunology, vol. 30, no. 1, pp. 1-6, 2014.

[50] M. Erol, O. Yigit, M. Tasdemir et al., "Potential of serum and urinary matrix metalloproteinase-9 levels for the early detection of renal involvement in children with Henoch-Schönlein purpura," Iranian Journal of Pediatrics, vol. 26, no. 4, p. e6129, 2016.

[51] C. Duymelinck, S. E. H. Dauwe, K. E. J. de Greef, D. K. Ysebaert, G. A. Verpooten, and M. E. de Broe, "TIMP-1 gene expression and PAI-1 antigen after unilateral ureteral obstruction in the adult male rat," Kidney International, vol. 58, no. 3 , pp. 1186-1201, 2000. 